therapy. Review. Ann Thorac Surg 2003; 75(6 Suppl):S29-35.

33 Hosenpud JD, Bennett LE, Keck BM, Boucek MM, Novick RJ. The Registry of the International Society for Heart and Lung Transplantation: seventeenth official report-2000. J Heart Lung Transplant 2000; 19:909-31.

\section{Suggested further reading}

1 SIGN Guideline 35: Diagnosis and treatment of heart failure due to left ventricular systolic dysfunction. Available on: http://www.sign.ac.uk/guidelines/fulltext/35/index.html

2 NICE Guideline. Chronic heart failure: Management of chronic heart failure in adults in primary and secondary care. Available from: http://www.nice.org.uk/

3 Remme WJ, Swedberg K; Task Force for the Diagnosis and Treatment of Chronic Heart Failure, European Society of Cardiology. Guidelines for the diagnosis and treatment of chronic heart failure. Eur Heart J 2001; 22:1527-60.

\section{Atrial fibrillation:}

\section{current perspectives}

Mark J Earley MRCP, Clinical Research

Fellow, Department of Cardiology,

St Bartholomew's Hospital, London

Simon C Sporton MD MRCP, Consultant Cardiologist, UCLH NHS Trust,

The Heart Hospital, London

Clin Med 2004;4:22-26

Dramatic advances in basic and clinical cardiac electrophysiology have rendered many arrhythmias amenable to cure by catheter ablation and have relegated anti-arrhythmic drugs to a subsidiary role. Unfortunately, however, the commonest sustained cardiac arrhythmia, atrial fibrillation (AF), remains incompletely understood and consequently difficult to manage. Nevertheless, important advances in our knowledge have recently been made; these will be reviewed in this article.

\section{Classification}

AF incorporates a range of subsets of which the mechanism and response to therapeutic intervention vary. A consensus on nomenclature has recently been achieved in an attempt to ensure appropriate management: ${ }^{1}$

- An AF event is either the first detected or a recurrent episode.

- Paroxysmal AF describes episodes that terminate spontaneously within seven days.

- $\mathrm{AF}$ is termed persistent if it lasts longer than seven days or requires cardioversion by any means to restore sinus rhythm.

- Permanent AF is the term used when cardioversion has failed or has not been attempted.

\section{Mechanisms}

\section{Arrhythmias}

The mechanism of virtually all tachyarrhythmias can be described as:

- re-entrant, where wavefronts of electrical activation propagate continuously around lines of electrical conduction block, or

- focal, where activation wavefronts spread from a discrete source of repetitive electrical discharge.

AF, however, consists of multiple, irregular, constantly varying wavefronts that cannot be easily analysed under physiological conditions in the human heart. As a consequence, much of our understanding has come from experimental studies in animal models and

\section{Key Points}

The ECG appearance of atrial fibrillation (AF) may result from different arrhythmia mechanisms

Electrical and structural remodelling are increasingly recognised as important influences on the natural history of AF and represent novel therapeutic targets

All patients with AF should be considered for anticoagulation with warfarin, depending on their stroke risk

Recent studies have failed to demonstrate an advantage of anti-arrhythmic drugs over palliation by ventricular rate control

Catheter ablation of the atrioventricular node is safe and gives good symptom relief when ventricular rate control cannot be achieved with drugs

Curative catheter ablation by pulmonary vein isolation is gaining popularity although numerous questions remain about the technique

KEY WORDS: atrial fibrillation, CPD, curative therapies, mechanisms, review 
caution is necessary in extrapolating these data to humans.

The source of these wavefronts can be a single re-entrant wavefront or an ectopic focus. The importance of this observation is that the focal origin provides a much simpler target for potentially curative radiofrequency ablation procedures in a proportion of patients.

\section{Remodelling and ion channels}

The natural history of paroxysmal AF is for the episodes to become more frequent, then persistent and ultimately permanent. ${ }^{2}$ AF per se induces changes in the atria that facilitate the development and maintenance of further episodes of AF. This process is termed remodelling. Electrical remodelling is caused by changes in the expression of atrial transmembrane ion channels, and in the long term can lead to fibrotic change (structural remodelling). The molecular biology of these ion channels is an area of intense research as they are subject to genetic variation that underlies familial $\mathrm{AF}$ in some cases and may also explain individual susceptibility to the development of AF. ${ }^{3}$ Furthermore, these channels represent a target for novel anti-arrhythmic drug strategies.

\section{Management}

\section{Anticoagulation}

Stroke prevention is a major aim of the treatment of all types of AF and is laid out clearly in international practice guidelines (Table 1). ${ }^{4}$ Both aspirin $(300 \mathrm{mg})$ and warfarin (international normalised ratio (INR) 2-3) prevent strokes, but the benefit of warfarin is greater especially in high-risk groups. ${ }^{5}$ Although ischaemic strokes may occur as a result of embolism from the left atrium, the pathogenesis is complex and the indication for anticoagulation should be determined by the overall stroke risk rather than the presence of $\mathrm{AF}$ at the time of assessment. Hence, no distinction should be made between paroxysmal, persistent and permanent AF. A therapeutic INR should be maintained for at least a month before and after planned

Table 1. American Heart Association/American College of Cardiology/European Society of Cardiology guidelines for the prescription of anticoagulation for patients with atrial fibrillation (AF) (adapted from Ref 4).

\begin{tabular}{ll} 
Patient & Therapy \\
\hline $\begin{array}{l}\text { Nor years old } \\
<00 \text { years old }\end{array}$ & No therapy or aspirin (325 mg) \\
$\begin{array}{l}\text { Heart disease } \\
\text { No risk factors for thromboembolism* }\end{array}$ & Aspirin (325 mg) \\
$>60$ years old & Aspirin (325 mg) \\
$\begin{array}{l}\text { Normal heart } \\
\text { No risk factors for thromboembolism* }\end{array}$ & \\
$>60$ years old & Warfarin (INR 2-3) \\
$\begin{array}{l}\text { Coronary disease or diabetes } \\
\text { No risk factors for thromboembolism* } \\
>75 \text { years old }\end{array}$ & \\
$\begin{array}{l}\text { Risk factors for thromboembolism* or } \\
\text { thyrotoxicosis (any age) } \\
\text { Rheumatic mitral valve disease }\end{array}$ & Warfarin (INR 2-3) \\
$\begin{array}{l}\text { Prosthetic valves } \\
\text { Persistent atrial thrombus on } \\
\text { echocardiography }\end{array}$ & Warfarin (INR 2.5-3.5 or higher) \\
\hline
\end{tabular}

* Risk factors for thromboembolism: symptomatic heart failure, left ventricular ejection fraction $<35 \%$, hypertension, previous thromboembolic event.

INR = international normalised ratio.

DC cardioversion. The decision whether or not to continue warfarin long term depends on the patient's overall stroke risk.

\section{Rate control versus rhythm control}

Several recent publications have ignited considerable debate about the value of restoring sinus rhythm. ${ }^{6-8}$ The Atrial Fibrillation Follow-up Investigation of Rhythm Management (AFFIRM) trial ${ }^{6}$ compared the strategy of rhythm control (using anti-arrhythmic drugs and DC cardioversion) with ventricular rate control alone. All-cause mortality was similar with either strategy, but the rhythm control group had a significantly higher rate of hospitalisation and adverse drug effects.

One interpretation of these results is that either strategy is acceptable. An alternative interpretation, however, is that both strategies are equally bad. Intuitively, restoration and maintenance of sinus rhythm should be better than palliation by ventricular rate control, but current anti-arrhythmic drugs are of limited efficacy and have considerable toxicity.

\section{Ventricular rate control}

The aim of ventricular rate control is to improve both cardiac function and symptoms. This is achieved in most cases with drugs that increase the refractory period of the atrioventricular (AV) node: digoxin, beta-blockers and calciumblockers such as verapamil and diltiazem. Although digoxin is useful for controlling the ventricular rate at rest, it has limited efficacy during conditions of sympathetic nervous system predominance including exertion. Beta-blockers and verapamil are more useful in these circumstances and a combination of drugs may be necessary to achieve adequate rate control. Assessment of rate control should include Holter monitoring and/or exercise testing.

In some patients ventricular rate control by drugs may not be achieved. In extreme cases a tachycardia-induced cardiomyopathy may develop. An effective solution is catheter ablation of the AV node, although this has disadvantages including dependency upon a permanent pacemaker. Survival is the same as with standard medical therapy ${ }^{9}$ and symptoms are greatly improved. Indeed, 

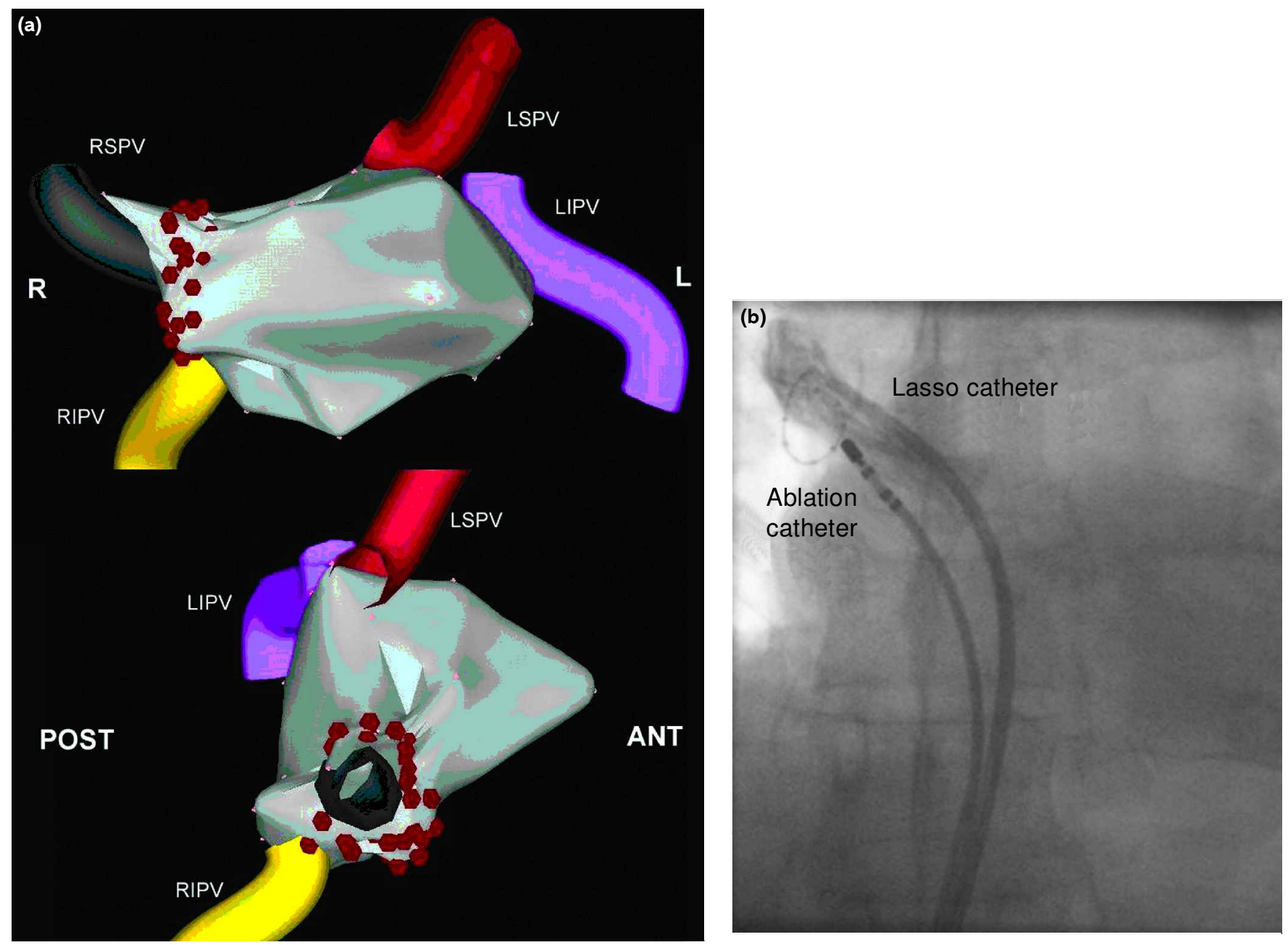

Fig 1. Comparison of an empirical anatomical approach with electrical isolation of the right pulmonary vein for the ablation of atrial fibrillation: (a) Empirical anatomical isolation of the right superior pulmonary vein (RSPV). An image of the left atrium and four pulmonary veins recorded with a Carto cardiac mapping system displayed in two views: anterior (ANT) (upper) and looking at the left atrium directly from the RSPV (lower) (four colour tubes = pulmonary veins; grey = body of the left atrium; red marks = individual lesions of radiofrequency ablation). A ring of lesions has been applied enclosing the RSPV, but well outside its ostium. There is no attempt to show that the vein is electrically isolated from the rest of the left atrium (LIPV = left inferior pulmonary vein; LSPV = left superior pulmonary vein; POST = posterior; R = right; RIPV = right inferior pulmonary vein). (b) Electrical isolation of the right superior pulmonary vein (RSPV). An X-ray image of the heart from the anteroposterior projection. Contrast has been injected into the RSPV. A 'Iasso' catheter with 10 electrodes is used to confirm whether the vein is electrically isolated from the rest of the left atrium. A steerable mapping/ablation catheter is used to deliver radiofrequency energy until electrical isolation of the pulmonary vein is achieved.

in patients with normal ventricular function, survival is identical to that of the general population. ${ }^{10}$

\section{Maintenance of sinus rhythm}

Device therapy. Specific situations in which pacemakers reduce the burden of AF are sick sinus syndrome and vagally-mediated AF. Aside from these clear indications, there are other novel pacemaker strategies to suppress AF. A comprehensive review of this interesting field has recently been published. ${ }^{11}$
A further innovation is the internal atrial defibrillator; this aims to prevent remodelling and lessen the tendency to develop persistent AF by detecting episodes of AF rapidly and restoring sinus rhythm through a low energy internal shock. Although effective, these devices are not popular due to the pain of repeated shocks.

Drug treatment. The evidence for anti-arrhythmic drugs for the maintenance of sinus rhythm has been comprehensively reviewed. ${ }^{4}$ Drugs with proven efficacy include flecainide, sotalol, propafenone and amiodarone. The choice of drug is largely governed by the presence of structural heart disease and comorbidity. Flecainide should be avoided in the presence of coronary artery disease or impaired left ventricular systolic function. Sotalol and propafenone are beta-adrenergic receptor antagonists and may therefore cause adverse effects related to that property. Although the efficacy of amiodarone is fractionally higher than that of the other anti-arrhythmic drugs, its side effect pro- 
Fig 2. A catheter maze for permanent atrial fibrillation. Basic geometry of the left atrium acquired using a non-contact mapping system is shown from the anterior (a) and posterior (b) projections (brown $=$ radiofrequency lesions of the basic catheter maze; highlighted in red $=$ ablation lines). From the posterior projection, the lines encircling the three ostia of the right pulmonary veins and the two ostia of the left pulmonary veins are highlighted. Line $\mathrm{T}$ is a line across the roof of the left atrium joining the right (RSPV) and left superior pulmonary veins (LSPV) (Inf = inferior; L = left; LIPV = left inferior pulmonary vein; $M V A=$ mitral valve annulus; RIPV = right inferior pulmonary vein; $\mathrm{R}=$ right; $\mathrm{RMPV}=$ right middle pulmonary vein; Sup = superior).

file should lead to caution over long-term use. Unfortunately, many patients with AF will remain symptomatic despite the administration of anti-arrhythmic drugs or be intolerant of them.

Curative catheter ablation. Based on the observation that ectopic foci in the pulmonary veins were responsible for some cases of AF and that by ablating these foci AF was curable, there has been a large increase in the number of catheter ablation procedures. ${ }^{12}$ Later it was recognised that multiple foci may coexist in one patient, and techniques have evolved to isolate all possible sources of ectopy (ie all four pulmonary veins, the coronary sinus and the venae cavae). Many aspects of this procedure remain contentious, in particular the need to prove electrical isolation of the pulmonary veins $^{13}$ or whether a purely empirical, anatomical approach is sufficient (Figs 1(a) and (b)). ${ }^{14}$ Published results are excellent using both approaches, but they are time-consuming and can be complicated by stroke and pulmonary vein stenosis. Numerous questions remain, including the identification of suitable patients and long-term safety and efficacy.

Permanent AF is not amenable to catheter ablation using the techniques described above as the dominant arrhythmia mechanism is multiple wavelet re-entry. To restore sinus rhythm

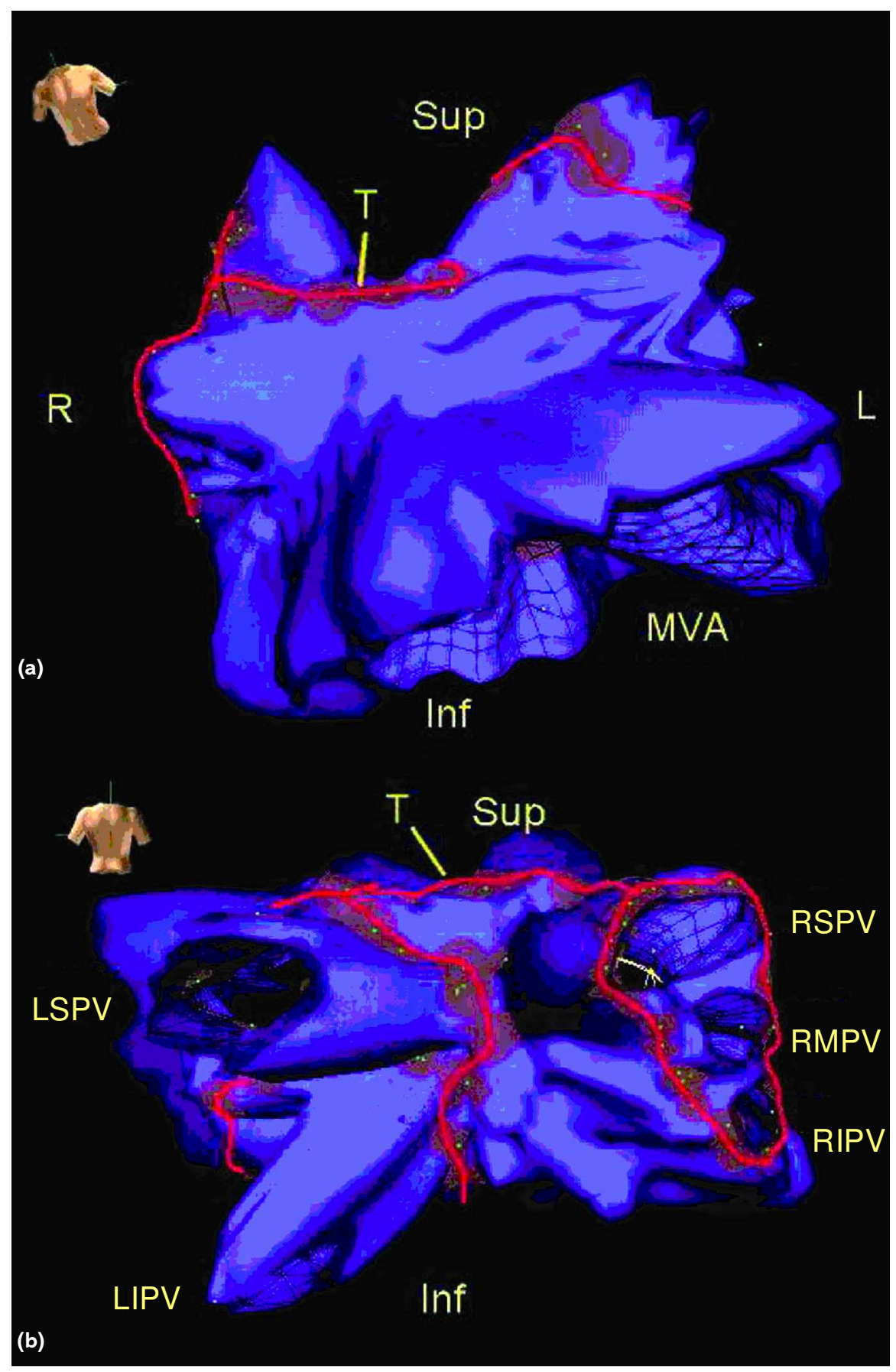

in these patients, a dramatic alteration of the atria is required so that the multiple re-entry wavefronts cannot be supported. This is achieved in the surgical maze, in which multiple lines of conduction block are created in both atria, dividing them into much smaller electrical compartments. This procedure is remarkably successful (99\% AF-free) ${ }^{15}$ but it is a major undertaking requiring cardiopulmonary bypass, with significant morbidity and mortality (2-3\%). The development of non-fluoroscopic catheter systems (eg non-contact mapping) and the success of ablation in paroxysmal AF has reawakened interest in a less invasive catheter maze procedure (Fig 2), bringing with it the prospect of an acceptable cure for this most intractable of arrhythmia. 


\section{References}

1 Levy S, Camm AJ, Saksena S, Aliot E et al. International consensus on nomenclature and classification of atrial fibrillation. J Cardiovasc Electrophysiol 2003;14:443-5.

2 Kopecky SL, Gersh BJ, McGoon MD, Whisnant JP et al. The natural history of lone atrial fibrillation. A population-based study over three decades. $N$ Engl $\mathrm{J} \mathrm{Med}$ 1987;317:669-74.

3 Nattel S. New ideas about atrial fibrillation 50 years on. Review. Nature 2002;415: 219-26.

4 Fuster V, Ryden LE, Asinger RW, Cannom DS et al. ACC/AHA/ESC Guidelines for the Management of Patients with Atrial Fibrillation. Circulation 2001;104:2118-50.

5 Connolly SJ. Preventing stroke in patients with atrial fibrillation: current treatments and new concepts. Review. Am Heart J 2003;145:418-23.

6 Wyse DG, Waldo AL, DiMarco JP, Domanski MJ et al; Atrial Fibrillation Follow-up Investigation of Rhythm Management (AFFIRM) Investigators. A comparison of rate control and rhythm control in patients with atrial fibrillation. N Engl J Med 2002;347:1825-33.

7 Van Gelder IC, Hagens VE, Bosker HA, Kingma JH et al. A comparison of rate control and rhythm control in patients with recurrent persistent atrial fibrillation. N Engl J Med 2002;347:1834-40.

8 Hohnloser SH, Kuck KH, Lilienthal J. Rhythm or rate control in atrial fibrillation - Pharmacological Intervention in Atrial Fibrillation (PIAF): a randomised trial. Lancet 2000;356:1789-94.
9 Wood MA, Brown-Mahoney C, Kay GN, Ellenbogen KA. Clinical outcomes after ablation and pacing therapy for atrial fibrillation: a meta-analysis. Circulation 2000; 101:1138-44.

10 Ozcan C, Jahangir A, Friedman PA, Patel PJ et al. Long-term survival after ablation of the atrioventricular node and implantation of a permanent pacemaker in patients with atrial fibrillation. $N$ Engl J Med 2001;344: 1043-51.

11 Lau CP. Pacing for atrial fibrillation. Review. Heart 2003;89:106-12.

12 Haïssaguerre M, Jaïs P, Shah DC, Takahashi A et al. Spontaneous initiation of atrial fibrillation by ectopic beats originating in the pulmonary veins. N Engl J Med 1998;339: 659-66.

13 Haïssaguerre M, Shah DC, Jaïs P, Hocini M et al. Electrophysiological breakthroughs from the left atrium to the pulmonary veins. Circulation 2000;102:2463-5.

14 Pappone C, Rosanio S, Oreto G, Tocchi M et al. Circumferential radiofrequency ablation of pulmonary vein ostia: a new anatomic approach for curing atrial fibrillation. Circulation 2000;102:2619-28.

15 Cox JL, Ad N, Palazzo T, Fitzpatrick S et al. Current status of the Maze procedure for the treatment of atrial fibrillation. Review. Semin Thorac Cardiovasc Surg 2000;12:15-9. 